\title{
Elevated circulating levels of succinate in human obesity are linked to specific gut microbiota
}

\author{
Carolina Serena ${ }^{1,2} \cdot$ Victoria Ceperuelo-Mallafré $^{1,2} \cdot$ Noelia Keiran $^{1,2} \cdot$ Maria Isabel Queipo-Ortuño $^{3,4} \cdot$ Rosa Bernal $^{4,5}$. \\ Ricardo Gomez-Huelgas ${ }^{4,5}$ - Mireia Urpi-Sarda ${ }^{6,7}$ - Mónica Sabater ${ }^{4,8}$ - Vicente Pérez-Brocal ${ }^{9,10}$. \\ Cristina Andrés-Lacueva ${ }^{6,7}$ - Andres Moya $\mathbb{1}^{9,10,11}$ - Francisco J Tinahones ${ }^{3,4}$ - Jose Manuel Fernández-Real ${ }^{4,8}$. \\ Joan Vendrell ${ }^{1,2,12} \cdot$ Sonia Fernández-Veledo ${ }^{1,2}$
}

Received: 6 October 2017 / Revised: 21 December 2017 / Accepted: 12 January 2018 / Published online: 12 February 2018

(c) The Author(s) 2018. This article is published with open access

\begin{abstract}
Gut microbiota-related metabolites are potential clinical biomarkers for cardiovascular disease (CVD). Circulating succinate, a metabolite produced by both microbiota and the host, is increased in hypertension, ischemic heart disease, and type 2 diabetes. We aimed to analyze systemic levels of succinate in obesity, a major risk factor for CVD, and its relationship with gut microbiome. We explored the association of circulating succinate with specific metagenomic signatures in cross-sectional and prospective cohorts of Caucasian Spanish subjects. Obesity was associated with elevated levels of circulating succinate concomitant with impaired glucose metabolism. This increase was associated with specific changes in gut microbiota related to succinate metabolism: a higher relative abundance of succinate-producing Prevotellaceae $(\mathrm{P})$ and Veillonellaceae $(\mathrm{V})$, and a lower relative abundance of succinate-consuming Odoribacteraceae $(\mathrm{O})$ and Clostridaceae $(\mathrm{C})$ in obese individuals, with the $(\mathrm{P}+\mathrm{V} / \mathrm{O}+\mathrm{C})$ ratio being a main determinant of plasma succinate. Weight loss intervention decreased $(\mathrm{P}+\mathrm{V} / \mathrm{O}+\mathrm{C})$ ratio coincident with the reduction in circulating succinate. In the spontaneous evolution after good dietary advice, alterations in circulating succinate levels were linked to specific metagenomic signatures associated with carbohydrate metabolism and energy production with independence of body weight change. Our data support the importance of microbe-microbe interactions for the metabolite signature of gut microbiome and uncover succinate as a potential microbiota-derived metabolite related to CVD risk.
\end{abstract}

\section{Introduction}

Cardiovascular disease (CVD) is a collective term used to describe heart and blood vessel disorders and constitutes the leading cause of death worldwide. In developed countries, CVD usually manifests as coronary artery disease, atherosclerosis, and hypertension, with central obesity playing an increasingly important role as a risk factor [1].

Carolina Serena and Victoria Ceperuelo-Mallafré contributed equally to this work.

Electronic supplementary material The online version of this article (https://doi.org/10.1038/s41396-018-0068-2) contains supplementary material, which is available to authorized users.

Joan Vendrell

jvo@comt.es

$\triangle$ Sonia Fernández-Veledo

sonia.fernandezveledo@gmail.com

Extended author information available on the last page of the article
Although much attention has focused on the generation of reactive oxygen species and consequent downstream ramifications in the progression of CVD [2], more studies are now centering on the human metabolome as a potential resource of biomarkers of CVD and also as novel therapeutic targets [3]. Against this background, the tricarboxylic acid intermediate succinate is concentrated in body fluids under conditions of hypoxia and inflammation where it serves as a universal metabolic signal of local stress and immunologic danger [4-6]. Accordingly, elevated levels of circulating succinate have been detected in several high-risk CVD states such as hypertension [7], ischemic heart disease [8], and type 2 diabetes mellitus (T2DM) [7, 9-11]. Under these scenarios, extracellular succinate is thought to signal through its cognate receptor SUCNR1/GPR91, with pathological implications in hypertrophic cardiomyopathy [8], obesity-related metabolic disturbances [12], renin-induced hypertension [10], and diabetic retinopathy [13].

There is a lack of consensus regarding the origin of circulating succinate. Although it is probable that damaged 
tissues contribute to the succinate found in circulation [13, 14], it is not unreasonable to expect that a relevant source of extracellular succinate might be attributed to intestinal efflux by specific gut microbiota, consistent with the ancestral bacterial origin of mitochondria. Traditionally described as a key intermediate in microbial propionate synthesis, succinate is a natural metabolic end product in some bacteria [15]. Remarkably, recent studies show that colonization of germ-free mice with the well-known succinate producer Prevotella copri increases cecal succinate, which improves glycemic control through activation of intestinal gluconeogenesis $[16,17]$.

Dysbiosis of the gut microbiota is associated with CVD and its traditional risk factors T2DM, insulin resistance, and obesity [15, 18-20]. Products of bacterial metabolism have been associated with both health benefits and disease. Thus, short chain fatty acids (SCFAs) produced via fermentation of nondigestible carbohydrates by colonic microbiota have been reported to enter systemic circulation $[15,21]$ and reach peripheral tissues, where they can activate their cognate receptors [21], improving body composition, glucose, and lipid homeostasis [22, 23]. Indeed, dietary SCFA supplementation was shown to decrease atherosclerotic lesions in murine models [24]. Conversely, other gut microbial-derived metabolites such as trimethylamine and trimethylamine $\mathrm{N}$-oxide (TMAO) are proatherogenic $[25,26]$ and have emerged as prognostic markers for incident cardiovascular events [27]. By analogy, we postulate that other organic acids such as succinate might be released by gut commensal bacteria into circulation, particularly in pathological conditions associated with an increase in intestinal permeability (leaky gut), such as obesity [28]. To the best of our knowledge, no studies have linked gut microbiota to circulating succinate. Here, we show that circulating succinate levels are increased in obesity and can be characterized by a specific intestinal bacterial signature. Weight loss alters the composition of succinate-producing and -consuming gut microbiota, which is coincident with a reduction in circulating levels of succinate. More importantly, spontaneous changes in intestinal succinate-metabolizing microbiota (producers vs. consumers) over a 2-year period are paralleled with changes in circulating succinate, with independence of weight loss, indicating a strong link between specific microbiome signature and blood succinate levels.

\section{Materials and methods}

\section{Study design and patients}

The present study comprised five different clinical substudies to serve the following different aims: (1) analyze circulating succinate levels in lean, obese, and diabetic subjects using a cross-sectional study, cohort I; (2) examine the relationship between gut microbiota and succinate (discovery cohort II and confirmatory cohort III); (3) establish a link between circulating succinate and gut microbiota (dietetic intervention study cohort IV and follow-up study cohort V).

All studies were conducted in accordance to the principles of the Declaration of Helsinki. All volunteers received information concerning their participation in the study and gave written informed consent. The studies were approved by the respective local ethics committee review boards of the participating Hospitals.

\section{Inclusion criteria for all subjects}

(1) Caucasian men and women; (2) body mass index (BMI) range from lean to obese (adequately represented in each group); (3) absence of underlying pathology on physical examination and tests other than those associated with an excess of weight or diabetes; and (4) signed informed consent for participation in the study.

\section{Exclusion criteria for all subjects}

(1) Serious systemic disease unrelated to obesity such as cancer, severe kidney, or liver disease; (2) systemic diseases with intrinsic inflammatory activity; (3) history of liver disease (chronic active hepatitis or cirrhosis) and/or abnormal liver function (alanine transaminase and/or aspartate transaminase three times above the upper normal value); altered renal function (creatinine $>1.4 \mathrm{mg} / \mathrm{dl}$ in women and $1.5 \mathrm{mg} / \mathrm{dl}$ in men); (4) pregnancy and lactation; (5) vegetarians or subjects subjected to irregular diet; (6) patients with severe disorders of eating behavior; (7) clinical symptoms and signs of infection in the previous month; (8) anti-inflammatory chronic treatment with steroidal and/or non-steroidal anti-inflammatory drugs; (9) prior antibiotic treatment in the last 3 months; (10) major psychiatric antecedents; and (11) uncontrolled alcoholism or drug abuse.

\section{Cross-sectional study cohort I}

\section{Design}

Observational single-point study.

\section{Participants}

Ninety-one subjects ( 49 women and 42 men) were included in the cross-sectional study (30 lean, 41 obese, and 20 patients with T2DM). Obesity was classified according to World Health Organization (WHO) criteria. Patients with T2DM were diagnosed according to American Diabetes Association criteria with a stable metabolic control in the 
previous 6 months, as defined by stable glycosylated hemoglobin values. No patient was insulin treated; $60 \%$ were taking metformin, $20 \%$ were treated with sulfonylurea and $<15 \%$ were treated with dipeptidyl peptidase- 4 inhibitors. Subjects were recruited at the Endocrinology Service at the University Hospital Joan XXIII (Tarragona, Spain).

\section{Intervention}

All patients had fasted overnight before collection of subcutaneous adipose tissue (SAT) and blood. SAT was obtained during scheduled non-acute surgical procedures including laparoscopic surgery for hiatus hernia repair or cholecystectomies. SAT samples were washed in phosphate-buffered saline (PBS) and immediately frozen in liquid $\mathrm{N}_{2}$ with storage at $-80{ }^{\circ} \mathrm{C}$, or used immediately for fractionation. For SAT fractionation, fresh SAT was diced into small pieces (10-30 mg), washed in PBS, and incubated in Medium 199 (Gibco, Grand Island, NY) plus 4\% bovine serum albumin and $2 \mathrm{mg} / \mathrm{ml}$ collagenase type I (Sigma-Aldrich, St. Louis, MO) for $1 \mathrm{~h}$ in a shaking water bath at $37^{\circ} \mathrm{C}$. Anthropometrical and clinical variables are summarized in Supplementary Table S1.

\section{Discovery cohort II}

\section{Design}

Observational single-point study.

\section{Participants}

Twenty female subjects were included in the cross-sectional study (10 lean and 10 obese). Obesity was classified according to WHO criteria. Subjects were recruited in the outpatient surgery at the Endocrinology Service at the University Hospital Virgen de la Victoria de Málaga (Malaga, Spain). The study participants received no antibiotic treatment, probiotics, prebiotics, or any other medical treatment influencing intestinal microbiota during the 3 months before the start of the study.

\section{Intervention}

All patients had fasted overnight before collection of blood and stool. Anthropometrical and clinical variables are summarized in Supplementary Table S2.

\section{Confirmatory cohort III}

\section{Design}

Observational single-point study.

\section{Participants}

Seventeen subjects ( 10 women and 7 men) were included in the study (9 lean and 8 obese). Obesity was classified according to WHO criteria. Subjects were recruited at the Endocrinology Service at the University Hospital Dr. Josep Trueta (Girona, Spain). The study participants received no antibiotic treatment, probiotics, prebiotics, or any other medical treatment influencing intestinal microbiota during the 3 months before the start of the study.

\section{Intervention}

All patients had fasted overnight before collection of blood and stool. Anthropometrical and clinical variables are summarized in Supplementary Table S2.

\section{Dietary intervention cohort IV}

\section{Design}

Intervention study.

\section{Participants}

Nine obese women (a subsample of the registered study ISRCTN88315555) were included in the study. Subjects were recruited in the outpatient surgery at the Endocrinology Service at the University Hospital Virgen de la Victoria de Málaga. The study participants received no antibiotic treatment, probiotics, prebiotics, or any other medical treatment influencing intestinal microbiota during the 3 months before the start of the study.

\section{Intervention}

Patients underwent an intervention involving a hypocaloric Mediterranean Diet and physical exercise program. The Mediterranean Diet included extra virgin olive oil and nuts and reduced the energy intake by approximately $600 \mathrm{kcal}$. The diet comprised fat $(35-40 \% ; 8-10 \%$ saturated fatty acids), carbohydrates (40-45\%; low glycemic index), and protein $(20 \%)[29,30]$. Adherence to the diet was measured as described previously [31]. Participants were encouraged to gradually increase their level of physical activity to reach at least $45 \mathrm{~min}$ per day over the course of the study, which was assessed by their personal trainer on a monthly basis. Participants kept a physical activity record using a GENEActiv@ accelerometer. Physical activity levels were evaluated using the Rapid Assessment of Physical Activity questionnaire [32].

Dietary and physical intervention involved individual visits with a nutritionist every week during the 3 months. 
Furthermore, a nutritional education program was initiated to modify dietary and lifestyle habits with the aim of promoting both weight loss and subsequent weight maintenance. All patients had fasted overnight before collection of blood and stool, before and after the intervention. Anthropometrical and clinical variables are summarized in Supplementary Table S3. None of the nine volunteers received antibiotic therapy, prebiotics, probiotics, synbiotics, vitamin supplements, or any other medical treatment influencing intestinal microbiota during the 3 months before the start of the study or during the study.

\section{Follow-up study cohort V}

\section{Design}

Spontaneous observational follow-up study.

\section{Participants}

Nineteen patients were followed for 2 years to evaluate the spontaneous evolution of the microbiota. General counseling was provided to the subjects. None of the 19 volunteers received antibiotic therapy, prebiotics, probiotics, synbiotics, vitamin supplements, or any other medical treatment influencing intestinal microbiota in the 3 months before the start of the study or during the study ( 2 years). All patients had fasted overnight before collection of blood and stool samples, before and after the follow-up period. Anthropometrical and clinical variables are summarized in Supplementary Table S4.

\section{Analytical determinations}

Blood samples were drawn after a 12-h fast. Serum/plasma was separated and immediately frozen at $-80{ }^{\circ} \mathrm{C}$. Serum biochemical parameters were measured in duplicate. Serum glucose, cholesterol, high density lipoprotein (HDL) cholesterol and triglycerides were measured by standard enzymatic methods (Randox Laboratories Ltd, Antrim, UK). Insulin was measured with an immunoradiometric assay (BioSource International, Camarillo, CA).

\section{Gene expression analysis}

Total RNA was extracted from SAT using the RNeasy Lipid Tissue Midi Kit (Qiagen, Hilden, Germany). Total RNA quantity was measured at $260 \mathrm{~nm}$ and purity was assessed by the OD260/OD280 ratio. For gene expression analysis, $1 \mu \mathrm{g}$ of RNA was reverse transcribed with random primers using the Reverse Transcription System (Applied Biosystems, Foster City, CA). For microRNA analysis, complementary DNA (cDNA) synthesis was performed with the TaqMan MicroRNA Reverse Transcription Kit (Thermo Fisher Scientific, Waltham, MA). Real-time PCR (qPCR) was conducted on a 7900HT Fast Real-Time PCR System using TaqMan Gene Expression Assays (Applied Biosystems) for ATGL (Hs 00386101_m1), ZAG (Hs 00426651_m1), ABHD5 (Hs01104373), HSL (Hs 00193510_m1), CD163 (Hs00174705_m1), HIF1A (Hs00153153_m1), IL1B (Hs001749097_m1), and CCL2 (Hs00234140_m1). Results were calculated using the comparative $\mathrm{Ct}$ method (2- $\Delta \Delta \mathrm{Ct})$ and expressed relative to the expression of the housekeeping gene $18 \mathrm{~S}$ (Hs 03928985_g1).

\section{Fecal microbiome analysis}

\section{S sequencing (cohort II and IV)}

Collected stool samples were immediately frozen at $-80^{\circ}$ C. Genomic DNA was extracted following the recommendations of the International Human Microbiome Standards (http://www.microbiome-standards.org) [33]. A frozen aliquot $(250 \mathrm{mg})$ of each sample was suspended in $250 \mathrm{ml}$ of guanidine thiocyanate, $40 \mathrm{ml}$ of $10 \% \mathrm{~N}$-lauroyl sarcosine, and $500 \mathrm{ml}$ of $5 \% \mathrm{~N}$-lauroyl sarcosine. DNA was extracted by mechanical disruption of the microbial cells with beads, and nucleic acids were recovered from clear lysates by alcohol precipitation. An equivalent of 1 $\mathrm{mg}$ of each sample was used for DNA quantification using a spectrophotometer (NanoDrop Technologies, Wilmington, DE). DNA integrity was examined by micro-capillary electrophoresis using an Agilent 2100 Bioanalyzer with the DNA $12,000 \mathrm{Kit}$, which resolves the distribution of double-stranded DNA fragments up to $17,000 \mathrm{bp}$ in length. Ribosomal 16S rRNA gene sequences were amplified from cDNA using the 16S Metagenomics Kit (Thermo Fisher Scientific, Italy). The kit included two primer sets that selectively amplify the corresponding hypervariable regions of the $16 \mathrm{~S}$ region in bacteria: primer set V2-4-8 and primer set V3-6, 7-9. The PCR conditions used were $10 \mathrm{~min}$ at $95^{\circ} \mathrm{C}, 30$ cycles of $30 \mathrm{~s}$ at $95{ }^{\circ} \mathrm{C}, 30 \mathrm{~s}$ at $58^{\circ} \mathrm{C}$, and $20 \mathrm{~s}$ at $72{ }^{\circ} \mathrm{C}$, followed by $10 \mathrm{~min}$ at $72{ }^{\circ} \mathrm{C}$. The concentration and the average size of each amplicon was determined using the Quant-iT PicoGreen dsDNA Assay Kit (Invitrogen); the amount of DNA fragments per microliter was calculated and libraries were created using the Ion Plus Fragment Library Kit (Thermo Fisher Scientific). Barcodes were added to each sample using the Ion Xpress Barcode Adapters 1-16 kit (Thermo Fisher Scientific). The library concentrations were determined using the Ion Universal Library Quantification Kit (Thermo Fisher Scientific). Emulsion PCR and sequencing of the amplicon libraries was performed on a Ion 520 chip (Ion $520^{\mathrm{TM}}$ Chip Kit) using the Ion Torrent S5 ${ }^{\mathrm{TM}}$ 
system and the Ion $520^{\mathrm{TM}} / 530^{\mathrm{TM}}$ Kit-Chef (Thermo Fisher Scientific) according to the manufacturer's instructions. After sequencing, the individual sequence reads were filtered using Ion Reporter Software V4.0 to remove low quality and polyclonal sequences.

\section{Metagenomic analysis (cohort III and V)}

Total DNA was extracted from frozen human stool samples using the QIAamp DNA Stool Mini Kit (Qiagen, Courtaboeuf, France). Quality assessment was performed with the prinseq-lite program applying the following parameters: min_length, 50, trim_qual_right, 20, trim_qual_type, mean; and trim_qual_window, 20. R1 and R2 reads from Illumina sequencing were joined using fastq-join from ea-tools suite. The fastq files were converted into fasta files using the 'fastq_to_fasta' tool from the FastXToolkit program. Those files were filtered against the human genome, downloaded from the NCBI FTP site (ftp://ftp.ncbi.nlm.nih.gov/genomes/H_sapiens/). The unaligned files, that is, those that did not map against the human genome, were the input files of a BLASTn search against a customized bacterial database (Bacteria_2015_06_09) consisting of the Human Microbiome and the bacterial genomes downloaded from the NCBI FTP site (ftp://ftp.ncbi.nlm.nih.gov/genomes/ HUMAN MICROBIOM/Bacteria/ and ftp://ftp.ncbi.nlm. nih.gov/ genomes/archive/old_refseq/Bacteria/). The best hits of the BLASTn output files were extracted, converted into contingency tables, and transformed into BIOM format to be used as input files of the Quantitative Insights Into Microbial Ecology (QIIME) open-source software pipeline version 1.9.0 [34, 35].

\section{Circulating succinate measurement}

\section{Fluorimetric method}

Circulating serum/plasma succinate levels were measured using the EnzyChromTM Succinate Assay Kit (BioAssay Systems, Hayward, CA). The assay sensitivity was $12 \mu \mathrm{M}$ and the intra- and interassay coefficients of variance were $<3.5$ and $6.95 \%$, respectively.

\section{LC-MS/MS and NMR analysis}

We validated circulating succinate levels obtained by the fluorimetric assay using liquid chromatography tandemmass spectrometry (LC-MS/MS) and nucelar magnetic resonance (NMR) analysis. To do this, a subsample of plasma samples from cohort I was prepared as previously reported with some modifications [36, 37]. Importantly, the concentration of succinic acid measured by the fluorimetric assay correlated with that measured by LC-MS/MS ( $r=$ $0.617, p=0.019)$ and by NMR $(r=0.769, p=0.043)$, indicating that we could use the fluorimetric assay to measure human succinate levels, which is faster and more economical than the other two methodologies.

\section{Circulating zonulin measurement}

We measured serum zonulin as a surrogate marker of intestinal permeability. Circulating plasma/serum zonulin levels were assessed using the Human Zonulin Elisa Kit (MyBiosource, San Diego, CA) [38, 39]. This assay has high sensitivity $(1 \mathrm{ng} / \mathrm{ml})$ and excellent specificity for detection of zonulin, and only detects the active (uncleaved) form. The intra- and interassay coefficients of variation for these determinations were $<10 \%$.

\section{Statistical analysis}

Statistical analysis was performed with the Statistical Package for the Social Sciences software, version 15 (SPSS, Chicago, IL). For clinical and anthropometrical variables, normally distributed data were expressed as mean \pm SD and for variables with no Gaussian distribution values were expressed as median $\left(25^{\text {th }}-75\right.$ th quartiles). Student's $t$-test with Bonferroni adjustment was used to compare the mean value of normally distributed continuous variables. For variables that did not have a Gaussian distribution, we used the Kruskal-Wallis test with post hoc Dunn's multiple comparison test. To analyze the differences in nominal variables between groups, we used the $\chi^{2}$ test. For microbiota data, statistical significance was tested by unpaired $t$-test or Mann-Whitney $U$-test as part of the SPSS software package. For intervention studies, Wilcoxon signed-rank test or paired $t$-test was used for paired analysis in the two prospective cohorts as appropriate. Pearson's and Spearman's correlation coefficients with Bonferroni adjustment were used to analyze the relationship between parameters. To determine which variables were associated with circulating succinate, multiple linear regression analyses were employed (stepwise forward selection procedures). All variables associated in the univariate analysis with succinate were included in their respective models. A $p$-value $<0.05$ was considered significant. For functional studies, statistical analysis was performed with $\mathrm{R}$ statistics software version 3.3.3. Wilcoxon rank-sum test was used for hypotheses testing analysis between the two groups (group 1 vs. group 2). Heat maps were generated using a hierarchical clustering algorithm to visualize the metagenomic function and metabolite differences within the data set. 

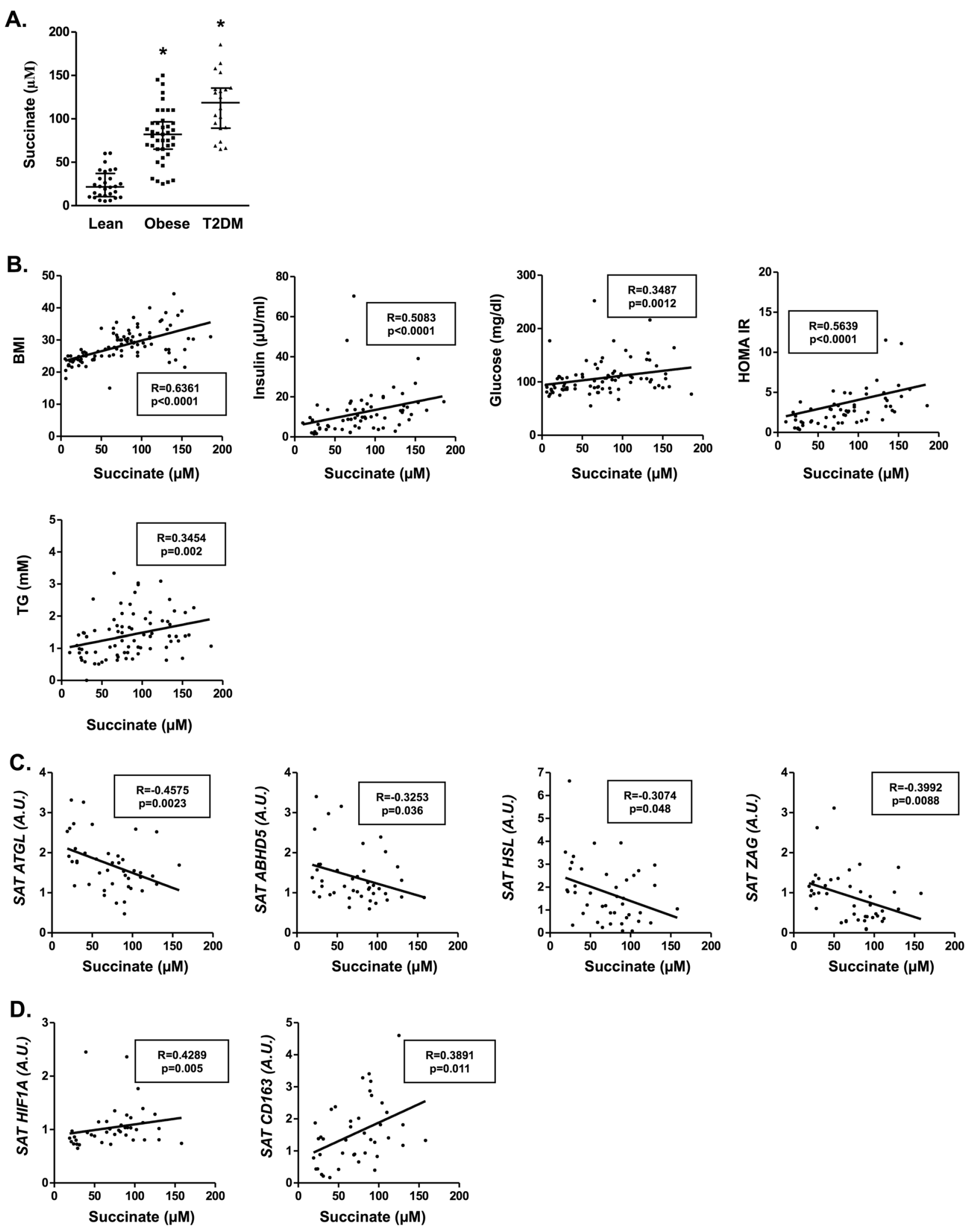
Fig. 1 Circulating succinate levels are increased in obesity and type 2 diabetes. a Circulating plasma levels in lean, obese and type 2 diabetes (T2DM) individuals. Data are expressed as median and interquartile range. Differences were analyzed by the Kruskal-Wallis test with post hoc Dunn's multiple comparison test. ${ }^{*} p<0.0001$ vs. lean. b Positive correlation between succinate levels and BMI, insulin, glucose, HOMA-IR, and triglycerides using the entire cohort. c Negative correlation between succinate levels and levels of SAT ATGL, SAT $A B H D 5$, SAT $H S L$, and SAT ZAG. d Positive correlation between succinate levels and SAT HIF1A and SAT CD163. SAT; subcutaneous adipose tissue. Statistical analyses for b-d: Spearman's correlation analysis. See also Table Supplementary S1 for all clinical characteristics of this cohort

\section{Results and Discussion}

\section{Circulating levels of succinate are elevated in obesity and associated with a worse metabolic profile}

In a cohort of 91 patients stratified according to obesity and T2DM (cohort 1), plasma succinate levels were significantly higher in obese than in lean individuals (Fig. 1a, Supplementary Table S1), and a comparable increase was detected in BMI-matched T2DM patients, in line with a recent report [11]. These results suggest that systemic succinate is also associated with body weight status. Accordingly, we found a positive association between circulating succinate levels and BMI (Fig. 1b), but also insulin, glucose, homeostasis model assessment of insulin resistance (HOMA-IR), and triglycerides (Fig. 1b). Consistent with the documented role of succinate in blood pressure regulation [7, 40], circulating succinate also correlated positively with diastolic blood pressure $(R=0.386, p=0.039)$. A multiple regression analysis model $\left(R^{2}=0.295\right)$ adjusted for age and gender showed that BMI and glucose $(\beta=$ $0.495 p<0.001$ and $\beta=0.279 p=0.013$, respectively) were the main determinants of circulating succinate levels.

Succinate has been shown to have antilipolytic actions in adipose tissue via engagement with SUCNR1, inhibiting the release of fatty acids from adipocytes [12, 41]. Consistent with this scenario, metabolic gene expression profiling in SAT from a representative subset of cohort I $(n=42)$ revealed a negative association between systemic succinate levels and genes encoding key enzymes involved in intracellular degradation of triacylglycerols, including adipose triglyceride lipase (ATGL), abhydrolase domain containing (ABHD5), and hormone-sensitive lipase (HSL) (Fig. 1c). A similar negative association was found for the gene encoding the secreted AT lipolytic factor zinc-alpha-2glycoprotein (ZAG) (Fig. 1c). Conversely, we found a positive association between succinate and hypoxiainducible factor $H I F-1 \alpha$ (Fig. 1d), a key transcription factor underlying chronic inflammation and AT dysfunction in obesity [42, 43]. Indeed, a clear function for succinate has been established in innate immune signaling, where it enhances interleukin-1 beta (IL-1 $\beta$ ) production via stabilization of HIF-1 $\alpha[6,44]$. Nevertheless, we found that systemic succinate levels were associated with the expression of the anti-inflammatory macrophage marker CD163 in SAT (Fig. 1d), but not with inflammatory markers such as IL-1 $\beta$ or MCP-1 $(R=0.116 p=0.466 ; R=0.039 p=$ 0.809 , respectively), supporting the notion that succinate might have differential intracellular and extracellular functions as previously noted for other stress-related factors such as osteopontin [45, 46] and heat shock proteins [47]. Of note, although some associations were also found in visceral adipose tissue, stronger correlations were detected in SAT, suggesting that the subcutaneous fat depot is more responsive to succinate than visceral fat.

\section{Gut microbiota composition is associated with circulating succinate levels}

In an independent cohort (cohort II, clinical, and anthropometrical characteristics are summarized in Supplementary Table S2), the serum concentration of succinate was significantly higher in obese than in non-obese individuals $(43.93 \pm 6.16 \mu \mathrm{M}$ vs. $23.2 \pm 1.57 \mu \mathrm{M}, p=0.0020)$. Of note, the concentration of succinate in serum is about onethird lower than that found in plasma ([13] and this study).

Analysis of gut microbiota composition by $16 \mathrm{~S}$ rRNA gene sequencing revealed an increase in the Firmicutes/ Bacteroidetes ratio in obese subjects (Supplementary Figure $1 \mathrm{~A}$ ), and decreased richness and biodiversity at the phylum and genus level (Supplementary Figure 1B-C) [4851]. We found that the relative abundance (RA) of Prevotellaceae $(37.52 \pm 3.86 \%$ vs. $12.93 \pm 3.97 \%, p=0.0005)$ and Veillonellaceae $(36.08 \pm 9.52 \%$ vs. $19.51 \pm 4.26 \%, p$ $=0.03$ ), known succinate producers [20, 52, 53], was higher in obese than in non-obese individuals (Fig. 2a). Accordingly, serum succinate levels positively correlated with Prevotellaceae $(R=0.465 ; p=0.039)$. Conversely, the RA of Odoribacteraceae $(1.58 \pm 0.68 \%$ vs. $6.18 \pm$ $1.64 \%, p=0.005)$ and Clostridaceae $(0.09 \pm 0.04 \%$ vs. $1.02 \pm 0.36 \%, p=0.05)$ families, known succinate consumers [54, 55], was significantly lower in obese than in non-obese individuals (Fig. 2a). No differences were detected in other bacterial families such as Paraprevotellaceae, Bacteroidaceae, or Ruminococcaceae, which are also related to succinate metabolism [52, 54, 5658]. Consequently, the ratio of (Prevotellaceae + VeillonellaceaelOdoribacteraceae + Clostridaceae $)(\operatorname{fam}(\mathrm{P}+\mathrm{V} /$ $\mathrm{O}+\mathrm{C})$ ), specific succinate producers per consumers, was significantly higher in obese subjects (Fig. 2b) and correlated positively with succinate serum levels (Fig. 2c). At the genus level, we found that the succinate-producing member Mitsuokella spp. was enriched in fecal samples of obese 
subjects $(9.67 \pm 5.37 \%$ vs. $0.11 \pm 0.11 \%, p=0.08)$, which was accompanied by a significant decrease in the succinateconsuming members Phascolarctobacterium spp. $(7.27 \pm$ $2.29 \%$ vs. $24.15 \pm 6.12 \%, p=0.018$ ) and Odoribacter spp. $(0.8 \pm 0.27 \%$ vs. $3.66 \pm 1.81 \%, p=0.017)$ (Supplementary Figure 1D). Correspondingly, the ratio of specific succinate producers/succinate consumers at the genus level was also significantly higher in obese than in non-obese individuals (Supplementary Figure 1E).

According to the "leaky gut" hypothesis, intestinal dysbiosis characteristic of obesity is directly related to translocation of bacteria and their products into systemic circulation [28]. As expected, circulating levels of zonulin, a useful biomarker of intestinal permeability [59-62], were significantly higher in obese than in non-obese individuals $(869.33 \pm 199.013 \mathrm{ng} / \mathrm{ml}$ vs. $500.87 \pm 44.61 \mathrm{ng} / \mathrm{ml}, \quad p=$ $0.04)$. A positive correlation was found between serum succinate and circulating zonulin $(R=0.61 ; p=0.011)$ (Fig. 2d), suggesting that akin to the elevated levels of circulating lipopolysaccharide in obesity [63, 64], intestinal permeability might be closely associated with the presence of succinate in systemic circulation.

To further investigate the relationship between serum succinate and the gut microbiome, we performed wholegenome shotgun sequencing of fecal DNA in an independent cohort (confirmatory cohort III; clinical and anthropometrical characteristics summarized in Supplementary Table S2). As noted in previous cohorts, succinate plasma levels were significantly higher in obese than in lean individuals $(101.72 \pm 9.37 \mu \mathrm{M}$ vs. $78.24 \pm 4.4 \mu \mathrm{M}, p=0.043)$. Furthermore, we detected a significant increase in the family Veillonellaceae $(2.37 \pm 0.39 \%$ vs. $1.41 \pm 0.24 \%, \quad p=$ 0.043 ) in obese subjects (Fig. 2e), as well as a positive correlation between Veillonellaceae and succinate levels in plasma $(R=0.773 ; p<0.001)$ (Fig. 2f). Accordingly, obese subjects had a higher $\operatorname{gen}(\mathrm{P}+\mathrm{V} / \mathrm{O}+\mathrm{C}$ ) ratio (Fig. $2 \mathrm{~g}$ ), which positively correlated with plasma succinate levels (Fig. 2h). Similar to cohort II, obese individuals had higher zonulin levels (Supplementary Table S2), which also positively associated with circulating succinate levels $(R=0.59$; $p=0.0152$ ).

Overall, these data demonstrate that despite the interindividual heterogeneity, circulating succinate levels are associated with specific components of gut microbiota. Interestingly, the microorganisms linked to circulating succinate levels have been previously related to CVD and/ or its risk factors. Thus, succinate-consuming genera such as Odoribacter and Clostridium have been linked to a decrease in clinical parameters associated with CVD risk $[65,66]$. By contrast, the Prevotella genus, which we found to be increased in obese individuals, has been recently associated with hypertension [67] and TMAO-induced atherosclerosis [68, 69]. Along these lines, Chen and colleagues have demonstrated that resveratrol modulates gut microbiota by inhibiting the Prevotella genus, which in turn induces a decrease in circulating TMAO levels [70], pointing to gut microbiota as an attractive target for pharmacological or dietary interventions to decrease the risk of developing CVD.

\section{Modification of gut microbiota by dietary weight loss intervention affects circulating succinate levels}

To determine whether diet-induced modifications in gut microbiota could be reflected in variations in circulating succinate levels, we carried out a prospective 12-week dietary intervention study in obese patients aimed to weight loss (cohort IV, Supplementary Table S3). Serum succinate levels decreased after the intervention (Fig. 3a) in parallel with an increase in genus and family richness (Supplementary Figure 2A). Although no significant differences were detected in genus or family diversity (Supplementary Figure 2B), we identified a decrease in the Firmicutes/ Bacteroidetes ratio (Supplementary Figure 2C), similar to that reported in a previous dietary weight loss intervention study [71-73].

In accordance with the results of the two previous cohorts (cohort II and III), we found a significant decrease in the succinate-producing families Prevotellaceae (17.91 $\pm 6.43 \%$ vs. $7.15 \pm 2.47 \%, p=0.019)$ and Veillonellaceae $(13.11 \pm 2.76 \%$ vs. $3.73 \pm 1.48 \%, p=0.027)$ after the dietary intervention (Fig. 3b). Comparable to that observed in cohort III, we found a positive correlation between the change in the incidence of Prevotellaceae ([Prevotellaceae] post-intervention $\left.-[\text { Prevotellaceae }]_{\text {basal }}\right)$ and succinate levels $(R$ $=0.751 ; p=0.019$ ) (Fig. 3c). Correspondingly, the fam $(\mathrm{P}$ $+\mathrm{V} / \mathrm{O}+\mathrm{C})$ ratio significantly decreased after weight loss (Fig. 3d) in parallel with a decrease in succinate, which was reflected in a positive correlation between the change in the $\operatorname{fam}(\mathrm{P}+\mathrm{V} / \mathrm{O}+\mathrm{C})$ ratio and the change in circulating succinate (post-intervention-basal) (Fig. 3e). Similar observations were found at the genus level (Supplementary Figure $2 \mathrm{D})$, and the $\operatorname{gen}(\mathrm{P}+\mathrm{V} / \mathrm{O}+\mathrm{C})$ ratio significantly decreased after the intervention (Supplementary Figure 2E).

Taken together, these results indicate that a short-term dietary weight loss intervention impacts different members of the gut commensal community related to succinate metabolism. Specifically, a decrease in succinate producers concomitant with an increase in succinate consumers at two taxonomic levels, which correlates with the decrease in systemic succinate levels observed, pointing to circulating succinate as a new dysbiosis-associated metabolite in the context of obesity.

Remarkably, joint analysis of both microbiota cohorts (cohort II and IV) validated the strong positive correlation between the $\operatorname{fam}(\mathrm{P}+\mathrm{V} / \mathrm{O}+\mathrm{C})$ ratio and circulating serum 


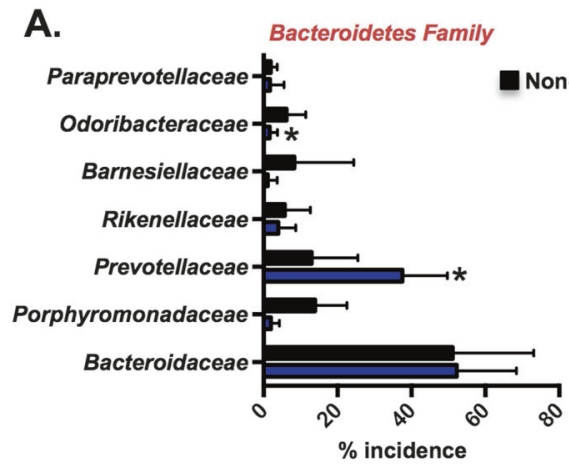

C.

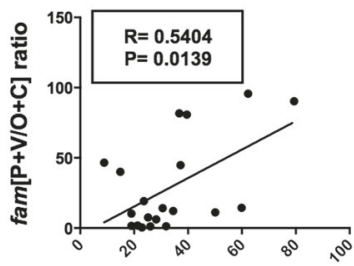

Succinate serum levels $(\mu \mathrm{M})$

F.

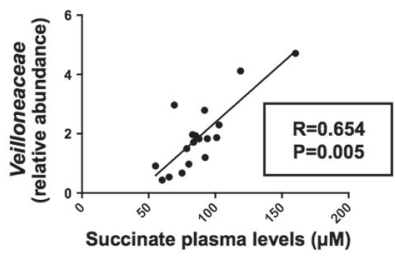

Non-obese

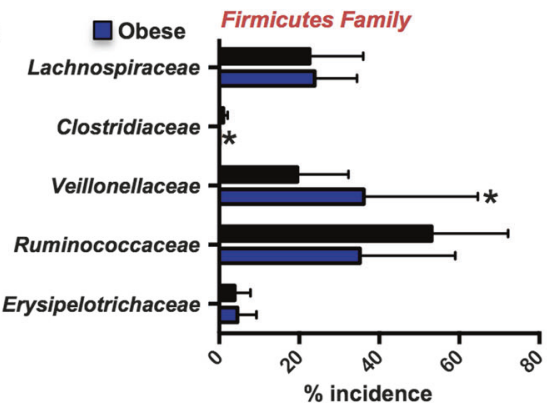

B.

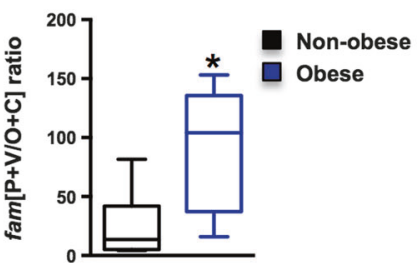

D.

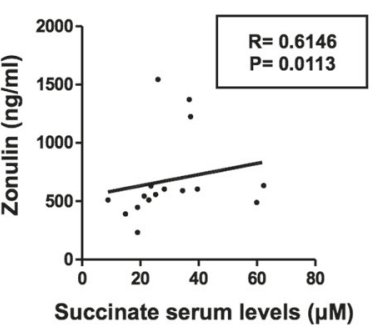

G.

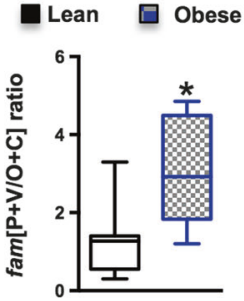

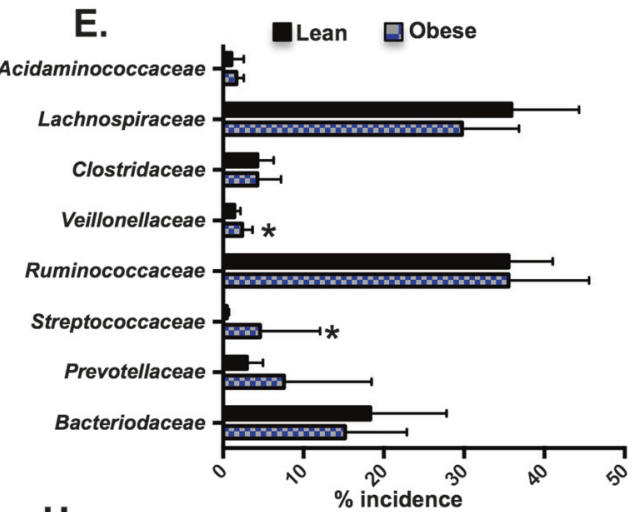

H.

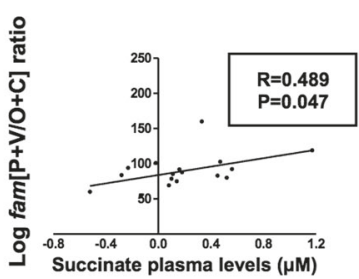

Fig. 2 Obese gut microbiota composition is associated with circulating succinate levels. a Percentage of incidence within Bacteroidetes and Firmicutes families in non-obese and obese individuals. b Differences between non-obese and obese individuals at the family level: families (Prevotellaceae plus Veillonellaceae/Odoribacteriaceae plus Clostridaceae $)(\operatorname{fam}(\mathrm{P}+\mathrm{V} / \mathrm{O}+\mathrm{C}))$ ratio. $\mathbf{c}$ Positive correlation between succinate serum levels and $\operatorname{fam}(\mathrm{P}+\mathrm{V} / \mathrm{O}+\mathrm{C})$ ratio. d Positive correlation between succinate serum levels and circulating zonulin levels. $\mathbf{e}$ Validation studies were performed using cohort III. Percentage of incidence within Bacteroidetes and Firmicutes families in lean and obese individuals. f Positive correlation between succinate plasma

succinate levels ( $n=38, R=0.646 ; p<0.001)$. Reassuringly, multiple regression analysis revealed that our proposed ratio based on (succinate-producing) vs. (succinateconsuming) families was the main determinant of systemic succinate levels $\left(R^{2}=0.744, \beta=0.597 ; p=0.007\right)$. Notwithstanding these strong correlations, exactly how microbial communities interact and use succinate is currently unknown. Moreover, other microbial groups could be responsible for succinate production (e.g., Succinovibrio spp., Ruminococcus spp., or Fibrobacter succinogenes) and consumption (e.g., Dialister spp., Phascolartobacterium succinatutes) $[52,54,56-58]$. Nevertheless, our results levels and Veilloneaceae. $\mathrm{g}$ Differences between lean and obese individuals in the $\operatorname{fam}(\mathrm{P}+\mathrm{V} / \mathrm{O}+\mathrm{C})$ ratio in the cohort III study. $\mathbf{h}$ Positive correlation between succinate serum levels and $\log \operatorname{fam}(\mathrm{P}+$ $\mathrm{V} / \mathrm{O}+\mathrm{C})$ ) ratio in the cohort III study. See also Supplementary Table S2 for all clinical characteristics of cohorts II and III. Data information: for $\mathbf{a}$ and $\mathbf{e}$, values are expressed as mean \pm SD. For $\mathbf{b}$ and $\mathbf{g}$, data are represented in box and whisker plot format (whiskers: $\min$ to $\max$ ). Statistical analyses: Mann-Whitney $U$-test. $* p<0.05$ vs. non-obese or lean. For c, d, f and h Spearman's or Pearson's correlation analysis with Bonferroni adjustment were used

strongly link the specific $\operatorname{fam}(\mathrm{P}+\mathrm{V} / \mathrm{O}+\mathrm{C})$ ratio to circulating succinate.

\section{Microbiota spontaneous evolution drives changes in systemic succinate}

Finally, to evaluate the spontaneous evolution of microbiota, we studied 19 subjects in whom general healthy habits counseling was provided: at baseline and at 2 years thereafter (see Materials and methods section, cohort V description in Supplementary Table S4). No significant differences in body weight were observed in these patients 
A.

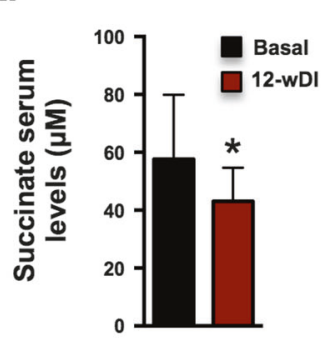

B.

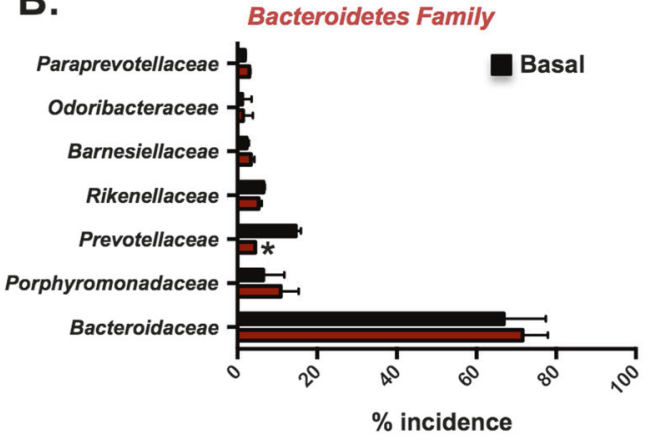

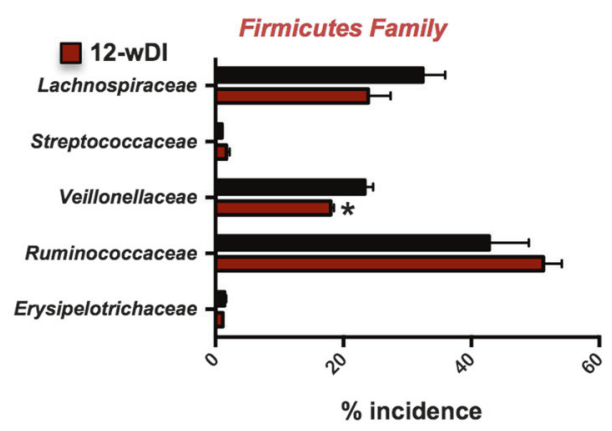

E.

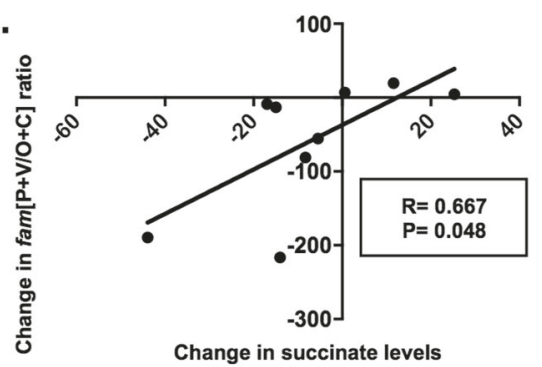

Fig. 3 Weight loss induced by dietary intervention modifies specific gut microbiota and impacts circulating succinate levels. a Circulating serum succinate levels in basal state and after a 12-week dietary intervention (12-wDI) from cohort IV. b Percentage of incidence within Bacteroidetes and Firmicutes families in obese individuals in basal state and after 12-wDI. c Positive correlation between the change in succinate serum levels (12-wDI[succinate]-basal[succinate]) and the change in Prevotellaceae (12-wDI [\% abundance Prevotellaceae]basal[\% abundance Prevotellaceae $]$ ). d Differences between basal

in the follow-up. We used a metagenomic approach rather than $16 \mathrm{~S}$ sequencing to analyze gut microbiota in this cohort. At the end of follow-up, subjects were classified into two groups in terms of changes in the ratio (succinateproducing) vs. (succinate-consuming) families (group 1, decreased ratio vs. group 2 , increased ratio). A reduction in $\operatorname{fam}(\mathrm{P}+\mathrm{V} / \mathrm{O}+\mathrm{C})$ was associated with a significant decrease in succinate levels (Table 1, group 1), whereas a significant increase in this ratio was related to a rise in systemic succinate (Table 1, group 2). These results show that variation in gut microbial composition independent of body weight changes are directly related to circulating succinate. Of note, elevated systemic succinate was paralleled with an impairment of glucose homeostasis, which contrasts with recently reported findings in animal models showing that microbiota-produced succinate is directly related to an improvement of glucose homeostasis [16]. Indeed, high succinate levels have been associated with various human pathological settings including CVD [8] and T2DM [7, 9-11].

Multivariate analyses identified statistically significant associations between the expression of 64 genes encoding metabolic enzymes, and the $\operatorname{fam}(\mathrm{P}+\mathrm{V} / \mathrm{O}+\mathrm{C})$ ratio. state and $12-\mathrm{wDI}$ in the $\operatorname{fam}(\mathrm{P}+\mathrm{V} / \mathrm{O}+\mathrm{C})$ ratio. e Positive correlation between the change in succinate serum levels (12-wDI[succinate]basal[succinate]) and the change in the $(12-w D I f a m(\mathrm{P}+\mathrm{V} / \mathrm{O}+$ C)-basal $\operatorname{fam}(\mathrm{P}+\mathrm{V} / \mathrm{O}+\mathrm{C})$ ) ratio. See also Supplementary Table S3 for all clinical characteristics of cohort IV. Data information: for $\mathbf{a}$ and b values are expressed as mean \pm SD. For d, data are represented in box and whisker plot format (whiskers: min to max). Statistical analyses: Wilcoxon signed-rank test. ${ }^{*} p<0.05$ vs. basal. For $\mathbf{c}$ and d, Spearman's correlation analysis with Bonferroni adjustment was used

Hierarchical clustering of these metagenomic data and the associations among $\operatorname{fam}(\mathrm{P}+\mathrm{V} / \mathrm{O}+\mathrm{C})$ ratio, circulating succinate and succinate-related microbial species, identified two clusters (labeled as A and B in Fig. 4a) with a clear relationship with the $\operatorname{fam}(\mathrm{P}+\mathrm{V} / \mathrm{O}+\mathrm{C})$ ratio (positive and negative correlations represented in green and red, respectively), which was mostly reflected by succinate levels. The metagenomic-derived clusters were also confirmed when associations with Prevotellaceae and Clostridaceae were analyzed, and a strong inverse relationship was detected. The main positive associations in cluster A were with genes encoding metabolic enzymes involved in amino-acid transport and metabolism ([E]), whereas cluster B showed a predominance of associations with genes related to energy production and conversion ([C]). Robust relationships with genes related to carbohydrate transport and metabolism ([G]) were revealed in both clusters. Interestingly, subclusters A1/A2 and B1/B2 were segregated on the basis of inverse associations with Veillonellaceae and Clostridaceae. These results link the $\operatorname{fam}(\mathrm{P}+\mathrm{V} / \mathrm{O}+\mathrm{C})$ ratio, specific gut microbiota and circulating succinate levels with a specific molecular entity and metabolic function. 
Table 1 Anthropometric and analytical characteristics in the cohort V

Follow-up study (cohort V)

\begin{tabular}{|c|c|c|c|}
\hline & Group 1 & Group 2 & $p$-Value \\
\hline$\Delta$ Ratio $\mathbf{P}+\mathbf{V} / \mathbf{C}+\mathbf{O}$ & $-1.14 \pm 1.29$ & $2.83 \pm 3.20$ & 0.002 \\
\hline$n$ & 8 & 11 & \\
\hline Sex (females/males) & $6 / 2$ & $4 / 7$ & 0.096 \\
\hline$\Delta$ Succinate $(\mathbf{m M})$ & $-25.19 \pm 27.11$ & $12.33 \pm 32.75$ & 0.017 \\
\hline$\Delta$ Weight $(\mathrm{kg})$ & $-0.25(-19.1$ to 3.47$)$ & $1.8(-1$ to 3.8$)$ & 0.351 \\
\hline$\Delta \mathrm{BMI}\left(\mathrm{kg} / \mathrm{m}^{2}\right)$ & $0.15(-3.35$ to 1.42$)$ & $0.30(-0.4$ to 1.3$)$ & 0.840 \\
\hline$\Delta$ Waist $(\mathrm{cm})$ & $8(-39$ to 11$)$ & $8(4.75$ to 10.5$)$ & 0.475 \\
\hline$\Delta \mathrm{Hip}(\mathrm{cm})$ & $2(-33$ to 6$)$ & $3(-0.5$ to 3.75$)$ & 0.887 \\
\hline$\triangle \mathrm{SBP}$ & $-0.71 \pm 23.73$ & $3.20 \pm 16.56$ & 0.693 \\
\hline$\triangle \mathrm{DBP}$ & $-1.71 \pm 13.74$ & $6.8 \pm 11.50$ & 0.185 \\
\hline$\Delta$ Glucose (mg/dl) & $-1 \pm 7.78$ & $6 \pm 6.15$ & 0.042 \\
\hline$\Delta$ Cholesterol (mg/dl) & $6.12 \pm 30.60$ & $-4.09 \pm 22.83$ & 0.415 \\
\hline$\Delta \mathrm{HDL}$ cholesterol $(\mathrm{mg} / \mathrm{dl})$ & $2.87 \pm 12.91$ & $0.55 \pm 7.12$ & 0.620 \\
\hline$\Delta$ Triglycerides $(\mathrm{mg} / \mathrm{dl})$ & $1.61 \pm 21.61$ & $6.63 \pm 27.56$ & 0.675 \\
\hline$\Delta \mathrm{Hb} 1 \mathrm{ac}(\%)$ & $0.41 \pm 0.70$ & $0.4 \pm 0.68$ & 0.969 \\
\hline$\Delta$ Prevotellaceae & $-3.66 \pm 4.29$ & $6.21 \pm 7.68$ & 0.005 \\
\hline$\Delta$ Veillonellaceae & $-0.44 \pm 1.89$ & $-0.7 \pm 1.38$ & 0.728 \\
\hline$\Delta$ Odoribacteraceae & ND & ND & - \\
\hline$\Delta$ Clostridaceae & $0.26(-0.66$ to 0.44$)$ & $-1.1(-2.82$ to -0.58$)$ & 0.039 \\
\hline
\end{tabular}

Data are presented as mean \pm SD or median $\left(25^{\text {th }}-75^{\text {th }}\right)$, as appropriate. Differences were analyzed by the unpaired $t$-test (normal distribution) or Mann-Whitney $U$-test (data not-normally distributed). Group 1 (patients ratio decreases at the end of follow-up) and Group 2 (patients ratio increases at the end of follow-up). A $p$-value $<0.05$ was considered significant

$B M I$ body mass index, $S B P$ systolic blood pressure, $D B P$ diastolic blood pressure, $N D$ not detected

Bold values represent those with statistically significant differences

The differences in the gene expression profiles associated with specific bacterial communities were also evident when we classified the cohort into two groups according the fam $(\mathrm{P}+\mathrm{V} / \mathrm{O}+\mathrm{C}$ ) ratio (group 1 vs. group 2) (Supplementary Figure $3 \mathrm{~A}$ ). An increase in the abundance of genes encoding enzymes associated with carbohydrate transport and metabolism ([G]), such as pectate lyase (EC:4.2.2.2), pectinesterase (EC:3.1.1.11) and glycosyl hydrolase (EC:3.2.1.52) after 2 years of follow-up, was detected in subjects in whom the $\operatorname{fam}(\mathrm{P}+\mathrm{V} / \mathrm{O}+\mathrm{C})$ ratio was increased in parallel with an increase in succinate levels. Curiously, a decrease in the abundance of genes encoding enzymes connecting the pentose phosphate pathway to glycolysis, such as ribulokinase (EC:2.7.1.16) and transaldolase (EC:2.2.1.2), was also observed in these patients. Genes associated with metabolic pathways linked to the biosynthesis of secondary metabolites ([Q]) such as succinylbenzoic acid-CoA ligase (EC:6.2.1.26), or those associated with amino-acid transport and metabolism ([E]) such as phosphoribosylformimino-5-aminoimidazole carboxamide ribotide isomerase (EC:5.3.1.16) and glutamate synthase (EC:1.4.1.14) were also modified. Intriguingly, all of these genes showed the strongest association with the $\operatorname{fam}(\mathrm{P}+\mathrm{V} / \mathrm{O}+\mathrm{C})$ ratio (Fig. 4a, enzyme names in red). More importantly, projection of these enzymes onto the Kyoto Encyclopedia of Genes and Genomes (KEGG) metabolic pathways map identified central metabolism as the main process associated with the $\operatorname{fam}(\mathrm{P}+\mathrm{V} / \mathrm{O}+\mathrm{C})$ ratio. Among them, glyocoside hydrolase and glutamate synthase were of particular interest because of their functional roles in glycolysis activation and succinate production via the GABA shunt pathway. Also worthy of mention was the negative association of $\operatorname{fam}(\mathrm{P}+\mathrm{V} / \mathrm{O}+\mathrm{C})$ ratio with ribulokinase and transaldolase, which also could promote glycolysis through inhibition of the pentose phosphate pathway (Fig. 4b). Mapping of the main enzymes positively or negatively correlated with $\operatorname{fam}(\mathrm{P}+\mathrm{V} / \mathrm{O}+\mathrm{C})$ ratio uncovered a clear connection between their functional features and succinate metabolism (adapted from KEGG metabolic pathways) (Supplementary Figure 3B).

In conclusion, our study reveals for the first time a strong association between microbial community, gene composition, and metabolism and circulating succinate levels in humans. Although it has been recently shown that 
Fig. 4 Associations of gut microbiota interactions, circulating succinate levels and metabolic bacterial functions. a Spearman's rank correlation between 64 genes encoding metabolic enzymes and fam $(\mathrm{P}+$ $\mathrm{V} / \mathrm{O}+\mathrm{C}$ ) ratio, circulating succinate levels, Prevotellaceae, Veillonellaceae, and Clostridaceae visualized as a heatmap. Annotation of heatmap: metabolic pathwaybased gene classification according to KEGG database; [C] Energy production and conversion; [E] Amino-acid transport and metabolism; [F] Nucleotide transport and metabolism; [G] Carbohydrate transport and metabolism; $[\mathrm{H}]$ Coenzyme transport and metabolism; [I] Lipid transport and metabolism; [P] Inorganic ion transport and metabolism and [Q] Secondary metabolites, biosynthesis and catabolism. b Positive and negative associations with the $\operatorname{fam}(\mathrm{P}+\mathrm{V} /$ $\mathrm{O}+\mathrm{C})$ ratio and metabolic enzymes as adapted from the KEGG metabolic pathways. See also Supplementary Table S4 and Table 1 for all clinical characteristics of cohort $\mathrm{V}$
A.

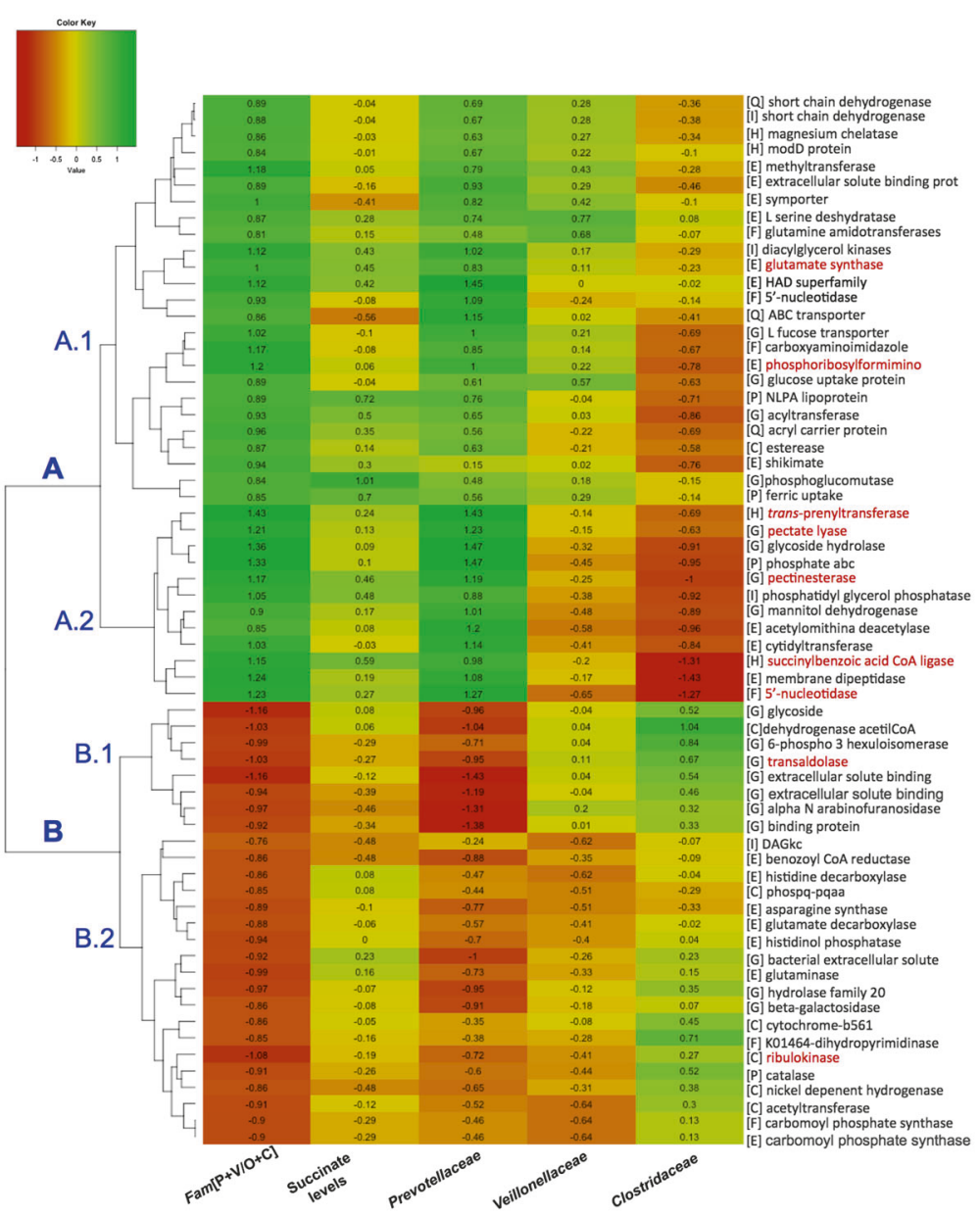

B.

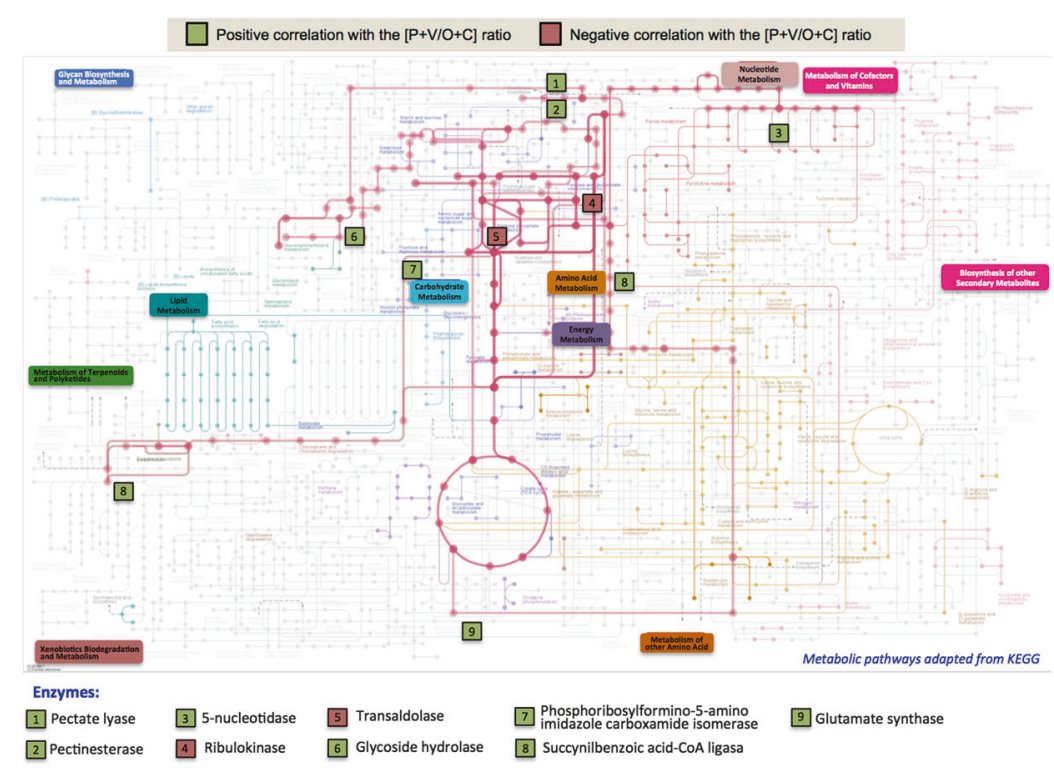

microbiota-derived succinate induces metabolic benefits by acting as an intestinal gluconeogenic substrate in germ-free mice [16], some caution should be exercised when extrapolating from mouse model data to humans. Moreover, a recent meta-analysis has suggested that in humans, the association between specific gut microbiota and obesity is 
smaller than that detected in mice and by most microbiome studies in humans [74]. Although the physiological effects of such modest differences are far from clear, they might become more apparent at the level of specific gene transcripts or metabolites. In this line, our study uncovers a clear association between succinate levels and obesityrelated metabolic disturbances, similar to that found in other CVD risk factors [7-11, 16, 75]. Clearly, although such an increase does not necessarily qualify succinate as a diseasecausing metabolite, its participation in the pathophysiology of obesity should not be entirely ruled out. Indeed, our data show a significant increase of glycemia in those patients who presented an increase in circulating succinate associated with changes in gut microbiota. Although engagement of succinate with its receptor has emerged as a link between metabolic stress and inflammation [4-6], it is still unclear whether succinate serves as a "harmful" signal or might have a protective role by acting as alarmin [76]. Furthermore, similar to what is seen with other microbial metabolites such as SCFAs, the beneficial and/or detrimental effects of succinate might depend on the amount synthesized [77, 78]. Although it is evident that further studies are required to prove causality, we hypothesize that obesity-related dysbiosis along with increased gut permeability might account for the higher levels of circulating succinate found in obese subjects. This may explain why colonization of germ-free mice with a probiotic succinate producer strain under normal conditions of intestinal permeability increases cecal, but not circulating, succinate levels [16]. Thus, we propose that modulation of $\operatorname{fam}(\mathrm{P}+$ $\mathrm{V} / \mathrm{O}+\mathrm{C})$ ratio in a pathological condition of increased intestinal permeability such as obesity will impact circulating succinate levels. Under this scenario, it should be considered that systemic succinate could act as a hormonelike metabolite, signaling through SUCNR1. How such levels impact host metabolism underlying obesity-related co-morbidities or whether manipulation of gut microbiota, specifically by increasing succinate-consuming families, might improve metabolic disturbances in obese patients are some of the open questions that should be explored in future work. Indeed, host or even dietary sources of succinate should not be dismissed. Our present findings point to succinate as a microbiota-derived metabolite with a potential role in obesity and metabolic-associated cardiovascular disorders, and strengthen the importance of microbial communities and their interactions when microbiota-derived bioactive compounds are studied.

Acknowledgements This study was supported by grants from the Spanish Ministry of Economy and Competitiveness (PI15/00143 to CS, PI12/01373 to RGH, PI15/01934 to JMF-R, SAF2015-65878-R to AM, PI14/00228 to JV and SAF2015-65019-R to SF-V), Instituto de Salud Carlos III (AC15/00022 to AM) and Generalitat Valenciana (project PrometeoII/2014/065 to AM), co-financed by the European
Regional Development Fund (ERDF). The Spanish Biomedical Research Center in Diabetes and Associated Metabolic Disorders (CIBERDEM) (CB07708/0012) and CIBERobn is an initiative of the Instituto de Salud Carlos III. CS acknowledges support from the "Ramón y Cajal' program from the Ministerio de Educación y Ciencia (RYC2013-13186), co-financed by the ERDF. SFV and MRBL acknowledges support from the Miguel Servet tenure-track program (CPII16/00008 and CP15/00028, respectively) from the Fondo de Investigación Sanitaria (FIS) co-financed by the ERDF. We also thank Dr. Kenneth McCreath for helpful comments on the manuscript.

\section{Compliance with ethical standards}

Conflict of interest The authors declare that they have no conflict of interest.

Open Access This article is licensed under a Creative Commons Attribution-NonCommercial-NoDerivatives 4.0 International License, which permits any non-commercial use, sharing, distribution and reproduction in any medium or format, as long as you give appropriate credit to the original author(s) and the source, and provide a link to the Creative Commons license. You do not have permission under this license to share adapted material derived from this article or parts of it. The images or other third party material in this article are included in the article's Creative Commons license, unless indicated otherwise in a credit line to the material. If material is not included in the article's Creative Commons license and your intended use is not permitted by statutory regulation or exceeds the permitted use, you will need to obtain permission directly from the copyright holder. To view a copy of this license, visit http://creativecommons.org/licenses/by-nc-nd/4.0/.

\section{References}

1. Vedanthan R, Fuster V. Urgent need for human resources to promote global cardiovascular health. Nat Rev Cardiol. 2011;8:114-7.

2. Yellon DM, Hausenloy DJ. Myocardial reperfusion injury. N Engl J Med. 2007;357:1121-35.

3. Mayr M. Metabolomics: ready for the prime time? Circ Cardiovasc Genet. 2008;1:58-65.

4. Rubic T, Lametschwandtner G, Jost S, Hinteregger S, Kund J, Carballido-Perrig $\mathrm{N}$, et al. Triggering the succinate receptor GPR91 on dendritic cells enhances immunity. Nat Immunol. 2008;9:1261-9.

5. Chouchani ET, Pell VR, Gaude E, Aksentijevic D, Sundier SY, Robb EL, et al. Ischaemic accumulation of succinate controls reperfusion injury through mitochondrial ROS. Nature. 2014;515:431-5.

6. Tannahill GM, Curtis AM, Adamik J, Palsson-McDermott EM, McGettrick AF, Goel G, et al. Succinate is an inflammatory signal that induces IL-1beta through HIF-1alpha. Nature. 2013;496:238-42.

7. Sadagopan N, Li W, Roberds SL, Major T, Preston GM, Yu Y, et al. Circulating succinate is elevated in rodent models of hypertension and metabolic disease. Am J Hypertens. 2007;20:1209-15.

8. Aguiar CJ, Rocha-Franco JA, Sousa PA, Santos AK, Ladeira M, Rocha-Resende C, et al. Succinate causes pathological cardiomyocyte hypertrophy through GPR91 activation. Cell Commun Signal. 2014;12:78.

9. Guo Y, Xie C, Li X, Yang J, Yu T, Zhang R, et al. Succinate and its G-protein-coupled receptor stimulates osteoclastogenesis. Nat Commun. 2017;8:15621.

10. Toma I, Kang JJ, Sipos A, Vargas S, Bansal E, Hanner F, et al. Succinate receptor GPR91 provides a direct link between high 
glucose levels and renin release in murine and rabbit kidney. $\mathrm{J}$ Clin Invest. 2008;118:2526-34.

11. van Diepen JA, Robben JH, Hooiveld GJ, Carmone C, Alsady M, Boutens L, et al. SUCNR1-mediated chemotaxis of macrophages aggravates obesity-induced inflammation and diabetes. Diabetologia. 2017;60:1304-13.

12. McCreath KJ, Espada S, Galvez BG, Benito M, de Molina A, Sepulveda $\mathrm{P}$, et al. Targeted disruption of the SUCNR1 metabolic receptor leads to dichotomous effects on obesity. Diabetes. 2015;64:1154-67.

13. Ariza AC, Deen PM, Robben JH. The succinate receptor as a novel therapeutic target for oxidative and metabolic stress-related conditions. Front Endocrinol (Lausanne). 2012;3:22.

14. Deen PM, Robben JH. Succinate receptors in the kidney. J Am Soc Nephrol. 2011;22:1416-22.

15. Sharon G, Garg N, Debelius J, Knight R, Dorrestein PC, Mazmanian SK. Specialized metabolites from the microbiome in health and disease. Cell Metab. 2014;20:719-30.

16. De Vadder F, Kovatcheva-Datchary P, Zitoun C, Duchampt A, Backhed F, Mithieux G. Microbiota-produced succinate improves glucose homeostasis via intestinal gluconeogenesis. Cell Metab. 2016;24:151-7.

17. Kovatcheva-Datchary P, Nilsson A, Akrami R, Lee YS, De Vadder F, Arora T, et al. Dietary fiber-induced improvement in glucose metabolism is associated with increased abundance of Prevotella. Cell Metab. 2015;22:971-82.

18. Nicholson JK, Holmes E, Kinross J, Burcelin R, Gibson G, Jia W, et al. Host-gut microbiota metabolic interactions. Science. 2012;336:1262-7.

19. Postler TS, Ghosh S. Understanding the holobiont: how microbial metabolites affect human health and shape the immune system. Cell Metab. 2017;26:110-30.

20. Vogt SL, Pena-Diaz J, Finlay BB. Chemical communication in the gut: effects of microbiota-generated metabolites on gastrointestinal bacterial pathogens. Anaerobe. 2015;34:106-15.

21. Koh A, De Vadder F, Kovatcheva-Datchary P, Backhed F. From dietary fiber to host physiology: short-chain fatty acids as key bacterial metabolites. Cell. 2016;165:1332-45.

22. Byrne CS, Chambers ES, Morrison DJ, Frost G. The role of short chain fatty acids in appetite regulation and energy homeostasis. Int J Obes (Lond). 2015;39:1331-8.

23. Puddu A, Sanguineti R, Montecucco F, Viviani GL. Evidence for the gut microbiota short-chain fatty acids as key pathophysiological molecules improving diabetes. Mediat Inflamm. 2014;2014:162021.

24. Aguilar EC, Santos LC, Leonel AJ, de Oliveira JS, Santos EA, Navia-Pelaez JM, et al. Oral butyrate reduces oxidative stress in atherosclerotic lesion sites by a mechanism involving NADPH oxidase down-regulation in endothelial cells. J Nutr Biochem. 2016;34:99-105.

25. Tang WH, Hazen SL. The contributory role of gut microbiota in cardiovascular disease. J Clin Invest. 2014;124:4204-11.

26. Wang Z, Klipfell E, Bennett BJ, Koeth R, Levison BS, Dugar B, et al. Gut flora metabolism of phosphatidylcholine promotes cardiovascular disease. Nature. 2011;472:57-63.

27. Li XS, Obeid S, Klingenberg R, Gencer B, Mach F, Raber L, et al. Gut microbiota-dependent trimethylamine $\mathrm{N}$-oxide in acute coronary syndromes: a prognostic marker for incident cardiovascular events beyond traditional risk factors. Eur Heart J. 2017a;38:814-24.

28. Slyepchenko A, Maes M, Machado-Vieira R, Anderson G, Solmi M, Sanz Y, et al. Intestinal dysbiosis, gut hyperpermeability and bacterial translocation: missing links between depression, obesity and type 2 diabetes. Curr Pharm Des. 2016;22:6087-106.
29. Davis C, Bryan J, Hodgson J, Murphy K. Definition of the Mediterranean diet: a literature review. Nutrients. 2015;7:9139-53.

30. Martinez-Gonzalez MA, Sanchez-Villegas A. The emerging role of Mediterranean diets in cardiovascular epidemiology: monounsaturated fats, olive oil, red wine or the whole pattern? Eur J Epidemiol. 2004;19:9-13.

31. Trichopoulou A, Costacou T, Bamia C, Trichopoulos D. Adherence to a Mediterranean diet and survival in a Greek population. N Engl J Med. 2003;348:2599-608.

32. Topolski TD, LoGerfo J, Patrick DL, Williams B, Walwick J, Patrick MB. The rapid assessment of physical activity (RAPA) among older adults. Prev Chronic Dis. 2006;3:A118.

33. Santiago A, Panda S, Mengels G, Martinez X, Azpiroz F, Dore J, et al. Processing faecal samples: a step forward for standards in microbial community analysis. BMC Microbiol. 2014;14:112.

34. Langmead B, Salzberg SL. Fast gapped-read alignment with Bowtie 2. Nat Methods. 2012;9:357-9.

35. Schmieder R, Edwards R. Quality control and preprocessing of metagenomic datasets. Bioinformatics. 2011;27:863-4.

36. Nagana Gowda GA, Gowda YN, Raftery D. Expanding the limits of human blood metabolite quantitation using NMR spectroscopy. Anal Chem. 2015;87:706-15.

37. Tulipani S, Llorach R, Urpi-Sarda M, Andres-Lacueva C. Comparative analysis of sample preparation methods to handle the complexity of the blood fluid metabolome: when less is more. Anal Chem. 2013;85:341-8.

38. Smecuol E, Sugai E, Niveloni S, Vazquez H, Pedreira S, Mazure R, et al. Permeability, zonulin production, and enteropathy in dermatitis herpetiformis. Clin Gastroenterol Hepatol. 2005;3:335-41.

39. Wang W, Uzzau S, Goldblum SE, Fasano A. Human zonulin, a potential modulator of intestinal tight junctions. J Cell Sci. 2000;113(Pt 24):4435-40.

40. He W, Miao FJ, Lin DC, Schwandner RT, Wang Z, Gao J, et al. Citric acid cycle intermediates as ligands for orphan G-proteincoupled receptors. Nature. 2004;429:188-93.

41. Regard JB, Sato IT, Coughlin SR. Anatomical profiling of G protein-coupled receptor expression. Cell. 2008;135:561-71.

42. Trayhurn P, Wang B, Wood IS. HIF-1alpha protein rather than mRNA as a marker of hypoxia in adipose tissue in obesity: focus on "inflammation is associated with a decrease of lipogenic factors in omental fat in women," by Poulain-Godefroy et al. Am J Physiol Regul Integr Comp Physiol. 2008;295:R1097. author reply R1098

43. Ye J. Emerging role of adipose tissue hypoxia in obesity and insulin resistance. Int J Obes (Lond). 2009;33:54-66.

44. Corcoran SE, O'Neill LA. HIF1alpha and metabolic reprogramming in inflammation. J Clin Invest. 2016;126:3699-707.

45. Inoue M, Shinohara ML. Intracellular osteopontin (iOPN) and immunity. Immunol Res. 2011;49:160-72.

46. Wang KX, Denhardt DT. Osteopontin: role in immune regulation and stress responses. Cytokine Growth Factor Rev. 2008;19:333-45.

47. Schmitt E, Gehrmann M, Brunet M, Multhoff G, Garrido C. Intracellular and extracellular functions of heat shock proteins: repercussions in cancer therapy. J Leukoc Biol. 2007;81:15-27.

48. Duncan SH, Lobley GE, Holtrop G, Ince J, Johnstone AM, Louis $\mathrm{P}$, et al. Human colonic microbiota associated with diet, obesity and weight loss. Int J Obes (Lond). 2008;32:1720-4.

49. Ley RE, Backhed F, Turnbaugh P, Lozupone CA, Knight RD, Gordon JI. Obesity alters gut microbial ecology. Proc Natl Acad Sci USA. 2005;102:11070-5.

50. Ley RE, Turnbaugh PJ, Klein S, Gordon JI. Microbial ecology: human gut microbes associated with obesity. Nature. 2006;444:1022-3. 
51. Zhang H, DiBaise JK, Zuccolo A, Kudrna D, Braidotti M, Yu Y, et al. Human gut microbiota in obesity and after gastric bypass. Proc Natl Acad Sci USA. 2009;106:2365-70.

52. Louis P, Hold GL, Flint HJ. The gut microbiota, bacterial metabolites and colorectal cancer. Nat Rev Microbiol. 2014;12:661-72.

53. Nakayama J, Yamamoto A, Palermo-Conde LA, Higashi K, Sonomoto K, Tan J, et al. Impact of westernized diet on gut microbiota in children on Leyte Island. Front Microbiol. 2017;8:197.

54. Ferreyra JA, Wu KJ, Hryckowian AJ, Bouley DM, Weimer BC, Sonnenburg JL. Gut microbiota-produced succinate promotes C. difficile infection after antibiotic treatment or motility disturbance. Cell Host Microbe. 2014;16:770-7.

55. Reichardt N, Duncan SH, Young P, Belenguer A, McWilliam Leitch C, Scott KP, et al. Phylogenetic distribution of three pathways for propionate production within the human gut microbiota. ISME J. 2014;8:1323-35.

56. Morotomi M, Nagai F, Sakon H, Tanaka R. Dialister succinatiphilus sp. nov. and Barnesiella intestinihominis sp. nov., isolated from human faeces. Int $\mathrm{J}$ Syst Evol Microbiol. 2008;58:2716-20.

57. O'Herrin SM, Kenealy WR. Glucose and carbon dioxide metabolism by Succinivibrio dextrinosolvens. Appl Environ Microbiol. 1993;59:748-55.

58. Watanabe Y, Nagai F, Morotomi M. Characterization of Phascolarctobacterium succinatutens sp. nov., an asaccharolytic, succinate-utilizing bacterium isolated from human feces. Appl Environ Microbiol. 2012;78:511-8.

59. Gulden E, Wong FS, Wen L. The gut microbiota and type 1 diabetes. Clin Immunol. 2015;159:143-53.

60. Mokkala K, Roytio H, Munukka E, Pietila S, Ekblad U, Ronnemaa $\mathrm{T}$, et al. Gut microbiota richness and composition and dietary intake of overweight pregnant women are related to serum zonulin concentration, a marker for intestinal permeability. J Nutr. 2016;146:1694-1700.

61. Moreno-Navarrete JM, Sabater M, Ortega F, Ricart W, Fernandez-Real JM. Circulating zonulin, a marker of intestinal permeability, is increased in association with obesity-associated insulin resistance. PLoS ONE. 2012;7:e37160.

62. Zak-Golab A, Kocelak P, Aptekorz M, Zientara M, Juszczyk L, Martirosian G, et al. Gut microbiota, microinflammation, metabolic profile, and zonulin concentration in obese and normal weight subjects. Int J Endocrinol. 2013;2013:674106.

63. Mokkala K, Pellonpera O, Roytio H, Pussinen P, Ronnemaa T, Laitinen K. Increased intestinal permeability, measured by serum zonulin, is associated with metabolic risk markers in overweight pregnant women. Metabolism. 2017;69:43-50.
64. Sun L, Yu Z, Ye X, Zou S, Li H, Yu D, et al. A marker of endotoxemia is associated with obesity and related metabolic disorders in apparently healthy Chinese. Diabetes Care. 2010;33:1925-32.

65. Karlsson FH, Fak F, Nookaew I, Tremaroli V, Fagerberg B, Petranovic D, et al. Symptomatic atherosclerosis is associated with an altered gut metagenome. Nat Commun. 2012;3:1245.

66. Tang WH, Kitai T, Hazen SL. Gut microbiota in cardiovascular health and disease. Circ Res. 2017;120:1183-96.

67. Li J, Zhao F, Wang Y, Chen J, Tao J, Tian G, et al. Gut microbiota dysbiosis contributes to the development of hypertension. Microbiome. 2017b;5:14.

68. Koeth RA, Wang Z, Levison BS, Buffa JA, Org E, Sheehy BT, et al. Intestinal microbiota metabolism of L-carnitine, a nutrient in red meat, promotes atherosclerosis. Nat Med. 2013;19:576-85.

69. Org E, Mehrabian M, Lusis AJ. Unraveling the environmental and genetic interactions in atherosclerosis: central role of the gut microbiota. Atherosclerosis. 2015;241:387-99.

70. Chen ML, Yi L, Zhang Y, Zhou X, Ran L, Yang J, et al. Resveratrol attenuates trimethylamine-N-oxide (TMAO)-induced atherosclerosis by regulating TMAO synthesis and bile acid metabolism via remodeling of the gut microbiota. MBio. 2016;7: e02210-02215.

71. Dao MC, Everard A, Clément K, Cani PD. Losing weight for a better health: role for the gut microbiota. Clin Nutr Exp. 2016;6:39-58.

72. Healey GR, Murphy R, Brough L, Butts CA, Coad J. Interindividual variability in gut microbiota and host response to dietary interventions. Nutr Rev. 2017;75:1059-80.

73. Cotillard A, Kennedy SP, Kong LC, Prifti E, Pons N, Le Chatelier $\mathrm{E}$, et al. Dietary intervention impact on gut microbial gene richness. Nature. 2013;500:585-8.

74. Sze MA, Schloss PD. Looking for a signal in the noise: revisiting obesity and the microbiome. MBio. 2016;7:pii:e01018-16

75. Correa PR, Kruglov EA, Thompson M, Leite MF, Dranoff JA, Nathanson MH. Succinate is a paracrine signal for liver damage. J Hepatol. 2007;47:262-9.

76. Rubic-Schneider T, Carballido-Perrig N, Regairaz C, Raad L, Jost $\mathrm{S}$, Rauld C, et al. GPR91 deficiency exacerbates allergic contact dermatitis while reducing arthritic disease in mice. Allergy. 2017;72:444-52.

77. den Besten G, van Eunen K, Groen AK, Venema K, Reijngoud DJ, Bakker BM. The role of short-chain fatty acids in the interplay between diet, gut microbiota, and host energy metabolism. J Lipid Res. 2013;54:2325-40.

78. Turnbaugh PJ, Ley RE, Mahowald MA, Magrini V, Mardis ER, Gordon JI. An obesity-associated gut microbiome with increased capacity for energy harvest. Nature. 2006;444:1027-31.

\section{Affiliations}

\section{Carolina Serena ${ }^{1,2} \cdot$ Victoria Ceperuelo-Mallafré $^{1,2} \cdot$ Noelia Keiran $^{1,2} \cdot$ Maria Isabel Queipo-Ortuño $^{3,4} \cdot$ Rosa Bernal $^{4,5}$. Ricardo Gomez-Huelgas ${ }^{4,5}$ - Mireia Urpi-Sarda ${ }^{6,7}$ - Mónica Sabater ${ }^{4,8}$ - Vicente Pérez-Brocal ${ }^{9,10}$. Cristina Andrés-Lacueva ${ }^{6,7}$. Andres Moya $\oplus^{9,10,11}$ - Francisco J Tinahones ${ }^{3,4}$ • Jose Manuel Fernández-Real ${ }^{4,8}$. Joan Vendrell ${ }^{1,2,12} \cdot$ Sonia Fernández-Veledo ${ }^{1,2}$}

1 Unitat de Recerca, Hospital Universitari de Tarragona Joan XXIII, Institut d’Investigació Sanitària Pere Virgili, Tarragona, Spain

2 CIBER de Diabetes y Enfermedades Metabólicas Asociadas (CIBERDEM), Instituto de Salud Carlos III, Madrid, Spain

3 Unidad de Gestión Clínica de Endocrinología y Nutrición,
Instituto de Investigación Biomédica de Málaga (IBIMA), Hospital Universitario de Málaga Virgen de la Victoria, Universidad de Málaga, 29010 Málaga, Spain

4 CIBER de Obesidad y Nutrición (CIBERObN), Instituto de Salud Carlos III, Madrid, Spain 
5 Servicio de Medicina Interna, IBIMA, Hospital Regional Universitario de Málaga, 29010 Málaga, Spain

6 Biomarkers and Nutrimetabolomics Laboratory, Nutrition, Food Science and Gastronomy Department, Faculty of Pharmacy and Food Science, University of Barcelona, Barcelona 08028, Spain

7 CIBER Fragilidad y Envejecimiento Saludable (CIBERFES), Instituto de Salud Carlos III, Madrid 28028, Spain

8 Department of Diabetes Endocrinology and Nutrition, Institut d'Investigació Biomèdica de Girona (IdIBGi), Girona, Spain
9 Genomics and Health Area, Foundation for the Promotion of Sanitary and Biomedical Research (FISABIO), València, Spain

10 CIBER de Epidemiology y Salud Pública (CIBERESP), Instituto de Salud Carlos III, Madrid, Spain

11 Institute of Integrative Systems Biology, Universitat de València, València, Spain

12 Universitat Rovira i Virgili, Tarragona, Spain 